\title{
A atenção básica à saúde do homem sob a ótica do usuário: um estudo qualitativo em três serviços do Rio de Janeiro
}

\author{
Primary healthcare for men from the user viewpoint: \\ a qualitative study in three services in Rio de Janeiro
}

\author{
Romeu Gomes ${ }^{1}$ \\ Lúcia Emília Figueiredo de Sousa Rebello ${ }^{1}$ \\ Elaine Ferreira do Nascimento ${ }^{1}$ \\ Suely Ferreira Deslandes ${ }^{1}$ \\ Martha Cristina Nunes Moreira ${ }^{1}$
}

\begin{abstract}
This paper seeks to analyze the male consumer viewpoint on primary healthcare services. The core objective is to find potential elements to underpin qualitative indicators of male user satisfaction. A qualitative approach involving semi-structured interviews with users was adopted, as well as studying medical records and ethnographic observation of three services in the municipality of Rio de Janeiro. The analysis was based on the method of interpretation of meanings, applying hermeneutic and dialectic principles. In general, users point to criteria to evaluate services positively, taking humanized care as a benchmark, based on communication and ability to offer swift care. The conclusion was that user recommendations involve demands primarily relating to failings in services to provide care specifically geared towards men.

Key words User satisfaction, Men's health, Men, Primary healthcare
\end{abstract}

Resumo O presente trabalho tem como objetivo analisar a ótica dos usuários sobre o atendimento prestado a homens no âmbito da atenção básica à saúde. Busca-se, principalmente, identificar possiveis elementos capazes de subsidiar a construção de indicadores qualitativos de satisfação de usuários masculinos. Utilizou-se a abordagem qualitativa, envolvendo entrevistas semiestruturadas com usuários, leitura de prontuários e observação etnográfica de três serviços do Município do Rio de Janeiro (RJ). A análise baseou-se no método de interpretação de sentidos, ancorandose em princípios hermenêuticos-dialéticos. Em geral, os usuários apontam critérios para avaliar positivamente os serviços, tomando como referências um atendimento humanizado, pautado na comunicação e capaz de oferecer prontidão no atendimento. Em termos de conclusão, constatouse que as recomendações dos usuários trazem demandas que se relacionam principalmente com as lacunas dos serviços para promover um atendimento especificamente voltado para homens.

Palavras-chave Satisfação de usuários, Saúde do homem, Homens, Atenção básica à saúde
${ }^{1}$ Instituto Fernandes Figueira, Fiocruz. Av. Rui Barbosa 716, Flamengo. 22250-020 Rio de Janeiro RJ.romeu@iff.fiocruz.br 


\section{Introdução}

Discussões nacionais e internacionais vêm sendo desenvolvidas para a promoção da atenção básica em saúde voltada para as especificidades da população masculina ${ }^{1}$. Nessas discussões, ressaltam-se, pelo menos, duas temáticas que se configuram como desafios para o sistema público de saúde: ações para que segmentos masculinos procurem os serviços de atenção básica em saúde e adequação dos serviços de saúde às demandas dos homens que procuram esses serviços. É nessa segunda temática que se circunscreve este estudo. Um dos caminhos para se chegar a essa adequação pode ser a escuta dos próprios usuários masculinos que buscam os serviços de saúde.

Essa escuta pode permitir conhecer a satisfação ou insatisfação desses usuários frente ao atendimento que lhes é prestado. As iniciativas de avaliação tomando como matéria-prima a perspectiva do usuário difundiu-se a partir dos anos 60 na Europa e EUA com um foco inicial na adesão ao tratamento. Nos anos de 1970 e 1980, essa prática passou a ser incorporada na avaliação da qualidade dos serviços de saúde ${ }^{2}$ e, no Brasil, ganha destaque a partir da segunda metade da década de 90.

Para além de constituir-se como indicador sensível da qualidade do serviço prestado, a satisfação dos usuários é uma categoria considerada estratégica para uma maior adequação dos serviços às suas demandas ${ }^{2-4}$. A satisfação pode ser entendida como percepção de qualidade quando se relaciona a um serviço prestado e não ao bem de consumo ${ }^{4}$. Na literatura, observa-se que o conceito de satisfação envolve um amplo espectro de fatores: estilo de vida, experiências prévias, expectativas de futuro, valores individuais e valores sociais. Essa categoria se remete a princípios relacionados a direitos individuais e de cidadania ${ }^{5}$.

Há estudos que, no lugar da expressão satisfação do usuário, utilizam a de paciente. No entanto, essa última costuma ser criticada porque pode ser associada à ideia de ausência de poder e de independência em relação ao terapeuta ${ }^{6}$. Ainda que pese as críticas acerca do termo paciente, destaca-se, dentre as referências teórico-metodológicas, o modelo denominado de Teoria Centrada na Satisfação do Paciente. Essa teoria sugere que o princípio da satisfação ou insatisfação, em geral, ocorre a partir dos nexos que se estabelecem entre a expectativa do paciente e a eficácia/ eficiência do atendimento. Nesse modelo, considera-se a satisfação do paciente com: $o$ atendimento inicial, o tempo de espera para esse aten- dimento e quem lhe presta o atendimento ${ }^{7,8}$. Esses aspectos servem de marco conceitual do presente trabalho.

$\mathrm{Na}$ revisão da literatura, realizada em novembro de 2008, a partir dos descritores de ciências da saúde (DeCS/MeSH) na base Medline, para o período compreendido entre 1997 e 2008, encontrou-se uma diferença quantitativa de artigos entre os descritores "satisfação do usuário" (5404 citações) e "satisfação do paciente"(30.616 citações). Esta diferença manteve-se ao serem associados os descritores "qualidade da assistência à saúde" (10 artigos) e "atenção básica à saúde" (1.510 artigos).

Refinando-se a busca com os descritores "saúde do homem" e/ou "homens" não se identificou nenhum artigo. Essa busca foi, ainda, ampliada, utilizando-se os mesmos descritores, aplicandoos ao método integrado de pesquisa BVS - Biblioteca virtual de Saúde.

Finalizada a busca, observou-se que, embora os estudos de satisfação dos usuários/pacientes em relação à qualidade da assistência à saúde nos serviços de atenção básica sejam numericamente significativos, em se tratando de usuários homens, a produção do conhecimento acessada na referida base encontra-se bastante reduzida. Nesse sentido, estudos acerca da temática em questão se fazem necessários para que se possa adequar esses serviços às demandas masculinas.

A partir dessa perspectiva o presente trabalho tem como objetivo analisar a ótica dos usuários sobre o atendimento prestado a homens no âmbito da atenção básica à saúde. Busca-se, principalmente, identificar possíveis elementos capazes de subsidiar a construção de indicadores qualitativos de satisfação de usuários masculinos.

\section{Material e Método}

O presente estudo é parte de uma pesquisa que objetivou investigar como se dá a relação homensassistência à saúde em cidades do Rio Grande do Norte, Pernambuco, Rio de Janeiro e São Paulo, financiada pelo Conselho Nacional de Desenvolvimento Científico e Tecnológico (CNPq), tendo como referência a proposta de avaliação a partir da triangulação de métodos ${ }^{9}$. Especificamente neste trabalho, em termos de referencial teóricometodológico, trabalhou-se com a perspectiva de construção de indicadores qualitativos, entendidos como aqueles que são construídos a partir da interpretação e julgamento dos próprios sujeitos envolvidos na avaliaçãa ${ }^{10}$. 
A pesquisa original foi submetida a distintos comitês de ética, sendo a vertente carioca do estudo submetida a dois com aprovação integral. Nesse município, o campo de pesquisa foi de três unidades públicas, sendo duas pertencentes ao Sistema Municipal de Saúde (aqui denominados de Serviço I e Serviço II) e um setor voltado para a saúde do adolescente que integra um instituto de ensino, pesquisa e assistência de referência do Sistema Único de Saúde - SUS (aqui denominado de Serviço III).

Tomando como base os princípios de amostra em pesquisa qualitativa, o foco incidiu em homens usuários, considerados em número suficiente para que fosse possível a saturação de sentidos, prevendo a possibilidade de haver inclusões sucessivas de sujeitos até que se conseguisse uma discussão densa das questões da pesquisa ${ }^{11}$.

Foram fixadas inicialmente 20 entrevistas para os serviços de atenção básica e 10 para os serviços que atendiam especificamente adolescentes. Em todos, utilizaram-se as seguintes faixas etárias: 15 a 25 anos, 26 a 45 e 46 a 65 .

Neste estudo, após a realização das 50 entrevistas, observou-se que o material era suficiente para a discussão das questões da pesquisa. Os entrevistados receberam nomes fictícios, iniciados com as letras M (Serviço I), V (Serviço II) e F (Serviço III) a fim de garantir o anonimato.

$\mathrm{O}$ instrumento de coleta privilegiado neste estudo foi a entrevista semiestruturada, realizada nos mencionados serviços, envolvendo principalmente os seguintes temas: Motivo do atendimento; Opinião sobre como deve ser o atendimento; Compreensão das informações fornecidas pelos profissionais; Identificações de possíveis dificuldades de atendimento e Recomendação para o serviço melhor atender ao usuário masculino. Os depoimentos gerados pelas entrevistas foram confrontados com a leitura dos respectivos prontuários e com os registros de observação.

$\mathrm{Na}$ caracterização dos homens entrevistados deste estudo, o quesito raça/etnia foi baseado nas denominações do Censo $2000^{12}$. Os demais foram classificados a partir de dados empíricos.

Em termos de tratamento qualitativo das informações, utilizou-se o método de interpretação de sentidos, baseando-se em princípios hermenêuticos-dialéticos que buscam interpretar o contexto, as razões e as lógicas de falas, ações e inter-relações entre grupos e instituições ${ }^{13}$, envolvendo os seguintes passos: (a) visão do conjunto e apreensão das particularidades do mate- rial da pesquisa; (b) identificação de sentidos atribuídos ao atendimento por parte dos entrevistados; (c) elaboração de indicadores qualitativos de bom atendimento a partir dos depoimentos; (d) comparação entre esses indicadores e as opiniões dos entrevistados acerca do atendimento a homens em geral; (e) diálogo comparativo entre as informações obtidas através dos depoimentos dos entrevistados, seus prontuários e notas de observação do atendimento; (f) elaboração de síntese interpretativa, procurando articular objetivo do estudo, marco teórico-conceitual de satisfação, dados empíricos e resultados de outros estudos.

\section{Sujeitos do Estudo}

Os dados sociodemográficos dos entrevistados dos serviços estudados apresentaram especificidades e se aproximam em alguns aspectos em relação ao conjunto dos usuários masculinos desses serviços. Em termos de faixa etária, por exemplo, não é possível fazer uma comparação entre os serviços, uma vez que o Serviço III só atende usuários até 18 anos de idade.

No que se refere à distribuição proporcional dos atendimentos realizados nos serviços de saúde, segundo sexo, nos serviços estudados verificou-se no período de 2002 a 2006 as seguintes médias de percentuais: 48,8\% (serviço I), 44,7\% (serviço II) e 42,9\% (serviço III). Estas, revelam que, assim como nos serviços de atenção básica em geral, a presença masculina tem se apresentado menor do que a feminina.

No conjunto dos 50 entrevistados (Tabela 1) predominam as seguintes características sociodemográficas: faixa etária de 46 a 65 anos (52\%), cor branca $(74 \%)$, ensino médio (46\%), estado civil solteiro $(48 \%)$ e responsável pela família (62\%). Sobressaem os homens mais maduros e com alta escolaridade, diferentemente dos serviços públicos em geral.

Em relação aos motivos que levaram os usuários a buscar os serviços predominaram os atendimentos relacionados a Diabetes e a Hipertensão, correspondendo a $20 \%$ e $21 \%$, respectivamente, dos 50 prontuários analisados. Observou-se que, em geral, os motivos se referiam a tratamento de doenças, indicando que esses sujeitos pouco ou nada procuravam por ações preventivas - no período da pesquisa - ratificando uma tendência ainda hegemônica do modelo curativo no perfil de utilização dos serviços. 
Tabela 1. Características sociodemográficas dos entrevistados

\begin{tabular}{|c|c|c|}
\hline $\begin{array}{l}\text { Características } \\
\text { sociodemográficas }\end{array}$ & $\mathrm{N}(\mathbf{5 0})$ & $\%$ \\
\hline \multicolumn{3}{|l|}{ Faixa etária } \\
\hline 15 a 25 anos & 12 & 24,0 \\
\hline 26 a 45 anos & 12 & 24,0 \\
\hline 46 a 65 anos & 26 & 52,0 \\
\hline \multicolumn{3}{|l|}{ Raça/cor (autorreferida) } \\
\hline Branca & 37 & 74,0 \\
\hline Parda & 9 & 18,0 \\
\hline Preta & 4 & 8,0 \\
\hline Indígena & 0 & 0 \\
\hline Amarela & 0 & 0 \\
\hline \multicolumn{3}{|l|}{ Escolaridade } \\
\hline Ensino fundamental & 20 & 40,0 \\
\hline Ensino médio & 23 & 46,0 \\
\hline Ensino superior & 7 & 14,0 \\
\hline \multicolumn{3}{|l|}{ Estado civil } \\
\hline Casado & 20 & 40,0 \\
\hline Divorciado & 2 & 4,0 \\
\hline Solteiro & 24 & 48,0 \\
\hline União estável & 4 & 8,0 \\
\hline \multicolumn{3}{|l|}{ Trabalho } \\
\hline Empregado & 24 & 60,0 \\
\hline Empresário & 1 & 2,5 \\
\hline Aposentado & 9 & 22,5 \\
\hline Desempregado & 3 & 7,5 \\
\hline Licença médica & 3 & 7,5 \\
\hline \multicolumn{3}{|c|}{ Pessoa responsável pela família } \\
\hline Sim & 31 & 62,0 \\
\hline Não & 15 & 30,0 \\
\hline Individual no domicílio & 4 & 8,0 \\
\hline
\end{tabular}

\section{Sentidos atribuídos ao bom atendimento}

Buscar compreender quais os critérios que os usuários utilizam para definir o que significa um bom ou mau atendimento constitui ação fundamental para entender as bases de sua satisfação acerca dos serviços ${ }^{2}$. Os depoimentos dos entrevistados revelaram alguns sentidos que servem de parâmetro para a construção de indicadores qualitativos de avaliação do atendimento que lhe é prestado ${ }^{10}$. Esses indicadores qualitativos são utilizados neste estudo para avaliar se os serviços estão conseguindo atender às demandas dos homens usuários, segundo a ótica dos próprios.

A partir dessa perspectiva, três sentidos atribuídos ao bom atendimento são focalizados a seguir. O primeiro deles nos traz como ideal o atendimento humanizado:
"Eu creio que a primeira coisa que deve se ter é um respeito pela pessoa humana, em muito dos casos não há esse respeito..." (Valério, 53 anos).

"Simpatia [...] a pessoa saber tratar o ser humano [...] do ser humano ser humano..." (Mário, 23 anos).

$\mathrm{O}$ atendimento humanizado na ótica dos usuários significa, principalmente, um atendimento "atencioso" e "respeitoso". Ainda que esse parâmetro se refira ao tratamento dispensado pelos serviços em geral é no médico que esse foco incide. Nesse sentido, se reivindica que a relação médico-usuário tenha como base uma boa relação interpessoal.

Os depoimentos que estruturam esse indicador se aproximam ou fazem eco da perspectiva da humanização a ser incorporada na área da saúde no sentido de situar os usuários dos serviços de saúde a partir do seu estatuto de sujeitos, articulando o uso das tecnologias com uma abordagem que parte da escuta, passando pela gerência dos afetos, para um comprometimento com a felicidade humana ${ }^{14}$.

Outro sentido é o atendimento ancorado na comunicação:

"Um atendimento [em] que haja oportunidade para o paciente poder relatar tudo aquilo que ele tá necessitando [...] e o profissional ouvir o paciente com paciência [...] uma comunicação [em] que os dois ficarão entendendo o que se passou ali naquela sala..." (Valfredo, 46 anos).

$\mathrm{O}$ atendimento ancorado na comunicação se traduz, na fala dos usuários, como aquele que se baseia na conversa e não se reduza ao ato de informar. Trata-se de uma conversa em que tanto o médico quanto o usuário ora fale ora escute, a partir de uma compreensão mútua. Essa comunicação pode ser estruturante num atendimento em que são envolvidos sujeitos pertencentes a segmentos com diferentes ethos, servindo de base para que haja um entendimento sobre as diferenças entre os critérios populares utilizados na avaliação da qualidade do atendimento e aqueles utilizados pelos profissionais. Pode ser o ponto de partida para uma abordagem antropológica em que se contextualizam as estruturas dos serviços de saúde, as circunstâncias socioeconômicas da vida dos usuários e as diferenças entre os modelos médicos e populares de saúde ${ }^{15}$.

A valorização do atendimento a partir da "humanização" e da "comunicação" - expressa nas falas dos homens entrevistados - caminha, de certa forma, na mesma direção de alguns estudos que abordam a qualidade do atendimento 
nos serviços de saúde utilizando o vínculo médico-paciente como indicador. Segundo Franco e Campos ${ }^{16}$, esses estudos têm enfocado que a satisfação dos pacientes se volta muito mais para o trato humanitário, a relação com o profissional e a informação recebida do que para o ato médico em si.

O terceiro sentido atribuído ao bom atendimento foi a prontidão do atendimento:

"O atendimento deve ser mais ágil possível, ou seja, você não precisar ficar horas e horas pra ser atendido [...] precisa ter vários profissionais à disposição [...] devia funcionar 24 horas [...] seria interessante o posto ter [...] laboratório [...] para fazer exames [...] mais básicos...” (Vitório, 36 anos).

"Você morre e você não é consultado. Então tem que ter esse intervalo de atendimento [menor], quer dizer, não [tanto tempo]. Marca hoje pra ser atendido em três meses, entendeu. Têm os exames, os exames também você marca [para] daqui três meses e pra pegar o resultado de um a dois meses..." (Valdomiro, 48 anos).

O indicador prontidão do atendimento estrutura-se principalmente a partir do desejo de que o tempo entre procura por atendimento e a efetivação do mesmo seja o menor possível. Talvez depois de sanado esse problema - a prontidão do atendimento tenha outro sentido, evoluindo para a aspiração de uma solução para o problema que originou a busca efetiva. Assim, do desejo da prontidão (em termos de redução do tempo em ser atendido) se caminha para a demanda de resolubilidade (solução do problema) ${ }^{17}$.

O problema da longa espera reflete uma realidade que ultrapassa os três serviços estudados, envolvendo uma reivindicação de um vasto segmento populacional. Uma avaliação da satisfação com a assistência de saúde prestada, com base na Pesquisa Mundial de Saúde, realizada no Brasil no ano de 2003, demonstrou que o tempo de espera foi o item que obteve menor grau de satisfação ${ }^{18}$.

Os três indicadores qualitativos de satisfação dos usuários com o atendimento prestado, elaborados a partir dos depoimentos dos nossos entrevistados, em geral se articulam direta ou indiretamente com a Teoria Centrada na Satisfação do Paciente ${ }^{7,8}$, bem como com taxonomias elaboradas para se avaliar a satisfação de usuários de serviços de saúde ${ }^{2}$. Vale mencionar que, de acordo com a Política Nacional de Humanização (PNH), humanizado é, entre outros aspectos, o atendimento ancorado: na escuta respeitosa, em processos comunicacionais e na resolutividade.
Assim, todos os três referenciais apontam para a humanização e não somente o primeiro.

Nesse sentido, as taxonomias elaboradas para a avaliação (visão técnica) e os sentidos subjacentes às falas dos nossos entrevistados (visão leiga) se aproximam, uma vez que destacam padrões de avaliação comuns. Assim, ao considerarem que o bom atendimento deve ser humanizado, ancorado na comunicação e na agilidade, os homens estudados estariam em concordância com os estudos de avaliação de satisfação de usuários de serviços de saúde.

\section{A ótica dos usuários \\ sobre o atendimento dos serviços}

Embora se espere que no campo representacional apareçam contradições, no acervo pesquisado houve uma coerência entre os parâmetros mencionados para o que é um bom atendimento e os depoimentos que avaliam o atendimento dos serviços. Dentro dessa lógica, os sentidos atribuídos pelos usuários a um bom atendimento humanizado, ancorado na comunicação e na prontidão do atendimento - serviram, espontaneamente, de padrão para esses sujeitos avaliarem como positivo ou como negativo o atendimento que receberam.

Entre os três serviços estudados, chama atenção àquele que tem um setor voltado especificamente para adolescentes (Serviço III), qualificado de forma extremamente positiva pelos entrevistados:

"Eu estava com dor [na região abdominal], eu acho que era um a infecção. E a doutora falou sempre que acontecesse alguma coisa, assim, casos especiais, não esperar a consulta não, vem aqui que eu te atendo [...] ligamos para o setor que faz a marcação, a gente chegou esperou e fui atendido, ela me examinou, fez os exames e me internou lá no $4^{\circ}$ andar, eu fiquei uma semana. Até as enfermeiras me atenderam bem..." (Flávio, 16 anos).

"Gosto [do atendimento] por causa de que eles tratam as pessoas muito bem aqui, deixam as pessoas muito livre para expor a sua opinião. É um lugar muito bom para ser atendido...” (Fúlvio, 16 anos).

A alta positividade por parte dos adolescentes talvez possa ser explicada pelo fato de o setor ser bem diferenciado no conjunto dos serviços, uma vez que pertence a um instituto de uma fundação federal, que articula assistência, pesquisa e ensino, através de cursos de pós-graduação stricto e lato sensu. 
Em relação aos Serviços I e II, as opiniões se dividem no momento em que os usuários avaliam o atendimento recebido. Quando esse atendimento é percebido como próximo ao modelo idealizado por eles, ainda que pesem as devidas ressalvas sobre algumas lacunas, ocorre uma avaliação positiva:

"O atendimento é bom, os médicos são atenciosos, as enfermeiras, o tratamento aqui é muito bom, eu não tenho o que reclamar [...] $\mathrm{O}$ único problema que está havendo no momento é sobre o remédio em casa [...] os correios não estão entregando o remédio em casa...” (Manuel, 60 anos)

"Gosto, gosto! Apesar dos problemas [...] eu gosto de ser atendido aqui [...] eu venho aqui e sou atendido" (Valmir, 47 anos).

Esses depoimentos trazem questões relacionadas a problemas que não são de responsabilidade direta dos serviços (como é o caso da não entrega dos remédios em domicílio), dificuldades de se equacionar a oferta dos serviços com a demanda de atendimento.

Por outro lado, essa avaliação positiva não quer dizer que houve necessariamente uma avaliação crítica acerca do atendimento, podendo expressar uma ausência de opinião ou mesmo uma aceitação passiva do atendimento que lhe prestado. Estudos de avaliação pontuam que a satisfação pode ser influenciada pela comparação com a precariedade de acesso vigente ${ }^{19}$.

Vaistman e Andrade ${ }^{6}$, com base em Willia$\mathrm{ms}$, apontam que a compreensão da visão dos usuários de serviços passa pelo o entendimento dos sentidos atribuídos por esses aos seus direitos e deveres na relação com o sistema de saúde. As autoras ressaltam ainda que, em se tratando de setor público, a complexidade dessa avaliação inclui elementos do imaginário social acerca de serviços públicos.

No conjunto dos depoimentos, as ideias de que o "atendimento particular é melhor" e que os contatos pessoais garantem melhor atendimento se destacam:

"Eu gostaria de atender num particular [...] mas [...] não tenho condição [...] no particular [...] o atendimento é rápido, eficiente..." (Mário, 23 anos).

"Olha... Se a pessoa pudesse ter condições de pagar uma consulta particular é claro que era melhor..." (Valmir, 47 anos).

"Aqui tudo [...] que eu preciso eu tenho [...] Eu trabalhava [aqui] com terceirizado na área de segurança [...] então eu peguei muitas informações $[. .$.$] fiz muitas amizades [...] essas amizades$ e informações faz com que eu chegue e fique a vontade e fique também numa boa, tranquilo e consigo aquilo que eu quero, em pouco tempo e no espaço que eu preciso, é o suficiente..." (Vilson, 45 anos).

"Gosto, até hoje não tenho que reclamar [...] apesar [de] que tem um pessoal que trabalha aqui, que me conhece, que também passa lá no meu trabalho, a gente se conhecemos aí fica mais fácil o meu atendimento, é por isso que eu gosto..." (Valdomiro, 48 anos).

$\mathrm{O}$ primeiro e o segundo depoimentos sugerem que o atendimento particular é melhor do que o público. Essa opinião tanto pode ter sido elaborada a partir da experiência do usuário com os dois tipos de atendimento como pode se ancorar no preconceito de que o particular é sempre melhor do que o público. Essa ideia é discutida em estudos realizados por Bós e Bós ${ }^{20}$ e Farias $^{21}$. Esses autores apontam que a intenção de buscar os serviços de saúde da rede privada está associada à ideia de uma maior segurança de atendimento em comparação à rede pública, principalmente quando há um maior comprometimento da saúde do usuário.

Já o terceiro e o quarto depoimentos indicam a existência da prática de um atendimento facilitado para aqueles que são conhecidos dos profissionais que atuam no serviço. Esse componente facilitador também pode ser identificado durante a observação participante nas unidades. Nos relatos informais de usuários beneficiados por este tipo de prática surge com um fator relevante na avaliação positiva de satisfação em relação ao serviço, fato que precisa ser questionado tanto em relação ao direito de acesso aos serviços quanto em relação à organização estrutural destes.

Os usuários que percebem o atendimento por eles recebidos como bem distante do seu modelo de ideal de atendimento, fazem uma avaliação negativa dos serviços:

"Tem médico que chega tarde [...] tem médico que não vem. O problema de reclamação deste hospital aqui é isso..." (Maurício, 40 anos).

"Em geral, amigo, acho que é muito deficiente aqui [...] o atendimento, porque é muito corrido [...] [O médico] não dá a atenção necessária que nós precisamos [...] com relação ao atendimento de funcionário [...] a má vontade $[\ldots]$ impera [...] É ruim porque a gente chega tudo sorrindo, querendo agradar, mas não tem jeito não ..." (Mateus, 53 anos)

"Sempre que eu posso, eu procuro ir ao hospital. Acho melhor [...] Uma das coisas que acho mais complicado em um atendimento, de um modo geral, é a agilidade..." (Vitório, 36 anos). 
$\mathrm{Na}$ avaliação negativa, como vemos nas falas dos usuários, pesou a demora para ser atendido e o acolhimento deficiente por parte dos profissionais. Nos demais materiais analisados, constatou-se que essa demora tanto se relaciona ao longo período de espera nas filas para a efetivação do atendimento quanto diz respeito ao longo intervalo entre a marcação de atendimento e a resolutividade do problema que gerou sua busca.

A insatisfação com o tempo de espera para se conseguir atendimento tem se destacado em várias pesquisas. A Pesquisa Mundial de Saúde sobre satisfação com a assistência ambulatorial e de internação, realizada no Brasil no ano de 2003, salientou o baixo grau de satisfação dos usuários entre todos os aspectos analisados ${ }^{22}$. Os depoimentos dos nossos entrevistados encontram ressonância com os dados referentes à satisfação dos usuários obtidos no processo avaliativo 2004-2006 do Programa Nacional de Avaliação dos Serviços de Saúde (PNASS). Esses dados demonstram que a população, após conseguir o acesso a um serviço de saúde, avalia de forma bastante positiva os serviços recebidos e que as maiores insatisfações dizem respeito ao tempo para a obtenção de um atendimento e ao tempo de espera dentro do hospital, além do tempo de consulta reduzido ${ }^{23}$.

Em geral, os entrevistados - quando questionados se indicariam ou não os serviços para outras pessoas - justificaram a sua indicação para outros usuários a partir da sua satisfação:

"[Indicaria] porque aqui [...] você é atendido muito bem [...] você pode expressar as suas opiniões [...] respeitam a gente...” (Fúlvio, 16 anos).

"Indicaria [o serviço] porque o acesso é fácil [...] os médicos são bons...” (Marcelo, 27 anos).

"Indicaria, como já indiquei, [...] porque aqui os médicos são bons [...] são atenciosos..." (Valentin, 49 anos)

Esses depoimentos apontam como motivos para a indicação, principalmente, o fato de o atendimento ser humanizado, baseado na comunicação e ter o acesso facilitado, aspectos já destacados como referência do bom atendimento. Por outro lado, essa positividade pode refletir uma certa conformidade diante de uma baixa expectativa que comumente ocorre frente aos serviços públicos de saúde ${ }^{23}$.

Em seus depoimentos, alguns usuários fizeram inúmeras recomendações para que os problemas do atendimento fossem sanados. Sugeriram - principalmente - o aumento da oferta de atendimento, uma maior atenção por parte do profissional, maior facilidade na marcação de consultas e melhor remuneração dos profissionais de saúde para que pudessem oferecer uma melhor assistência.

Entre as reivindicações dos usuários, destacam-se, em específico, recomendações para um melhor atendimento do usuário homem:

"Na parte, por exemplo, de urologia, não tem especialista nessa parte e a parte de sexologia também, de problemas ligados à impotência, disfunção erétil, essas coisas que não parece, mas [risos] atormenta bastante os homens [...] Eu não encontrei serviço especializado pra isso. Eu encontrei até médico que me orientava e tal, mas não um especialista, uma sexóloga, um psicólogo [...] Então, se o homem precisar ele não tem...” (Vitório, 36 anos).

"Para atender os usuários homens poderia ter [...] uma reunião, pra falar das doenças que os homens pode ter, que pode não ter [...] passar mais informações pro homem, pra ele ter consciência assim que não é porque ele tem vergonha, [que ele vai deixar] de fazer o exame de próstata..." (Valdomiro, 48 anos).

"Creio que a saúde pública é muito mais voltada pra saúde da mulher, então preparar pessoas mais voltadas pra [...] a saúde do homem...” (Valério, 53 anos).

"Em primeiro lugar o incentivo, o incentivo nas propagandas, televisão, campanhas, cartazes [...] Não [...] só em termos, por exemplo, da próstata, mas outros quadros [...] no sentido de mostrar de que é importante ficar vivo sim, é importante ir procurar as pessoas para nos ajudar..." (Valfredo, 46 anos).

"Talvez melhorasse um pouco [se tivesse] atendimento reservado só para homens, separado..." (Valmir, 47 anos).

As recomendações voltadas para o atendimento de homens caminham na direção da oferta de um atendimento que vá ao encontro das especificidades masculinas. Dentro dessa lógica, são recomendadas campanhas, reuniões de esclarecimentos, a existência de setores separados do atendimento a mulheres e crianças. No que se refere a especialidades médicas, o atendimento de urologia é reivindicado, revelando que não há igual preocupação, como nos casos das mulheres, através do acompanhamento ginecológico.

Interessante observar que as recomendações que os homens fizeram para um melhor atendimento de segmentos masculinos reforçam as explicações dadas por outros homens que não estavam nos serviços de saúde, envolvidos numa pesquisa também realizada no Município do Rio de Janeiro ${ }^{24}$. Os entrevistados dessa outra pes- 
quisa apresentavam os seguintes motivos para o fato de os homens não procurarem os serviços de saúde: os serviços eram mais voltados para mulheres e crianças, os homens em geral tinham vergonha de se expor, não havia um setor só para homens serem atendidos e não foram educados para se cuidarem.

\section{Considerações Finais}

Os depoimentos dos usuários homens que, em geral, não se diferenciaram por faixas etárias, não expressaram necessariamente uma perspectiva de gênero. Tanto as concepções de um bom atendimento quanto a avaliação do atendimento em si revelaram questões que podem servir para a discussão da satisfação de homens e mulheres.

Entretanto, no que se refere às recomendações, observa-se que estas trazem demandas que se relacionam principalmente a lacunas dos serviços para promover um atendimento especifi- camente voltado para homens, revelando que deve haver um melhor preparo tanto por parte do usuário como do profissional. Em outras palavras, o usuário deve ser preparado para ficar mais à vontade para apresentar as suas demandas de cuidados e, em contrapartida, os profissionais devem ser melhor formados para atendêlo. Essa melhor formação profissional, por sua vez, deve se vincular a "mudanças nos aspectos gerenciais e organizacionais dos serviços de saúde, assim como nas estruturas físicas, para articular avanços tecnológicos e acolhimento, melhores condições de trabalho e processos comunicacionais" 25 .

Por último, constata-se a necessidade de se realizar outros estudos voltados para a percepção de homens usuários da atenção básica sobre o atendimento a eles prestados, uma vez que, na literatura, esses estudos são praticamente inexistentes, em termos de realidade nacional. Nesse sentido, a discussão aqui desenvolvida constituise num estudo exploratório sobre o tema.

\section{Colaboradores}

R Gomes, LEFS Rebello, EF do Nascimento, SF Deslandes, MC Moreira participaram igualmente de todas as etapas da elaboração do artigo. 


\section{Referências}

1. Schraiber LB, Gomes R, Couto MT. Homens na pauta da saúde coletiva. Cien Saude Colet 2005; 10(Supl. 1):7-17.

2. Esperidião M, Trad LBA. Avaliação de satisfação de usuários. Cien Saude Colet 2005; 10(Supl. 1):303312.

3. Bosi ML, Affonso KC. Participação popular e saúde: com a palavra, os usuários da rede pública de serviços. Cad Saude Publica 1998; 14(Supl. 2):355-365.

4. Castillo L, Dougnac A, Vicente I, Muñoz V, Roja V. Los predictores de satisfacción de pacientes en un centro hospitalario universitario. Rev Méd Chile 2007; 135(6):696-701.

5. Bosi MLM, Affonso KC. Cidadania, participação popular e saúde: com a palavra, os usuários da Rede Pública de Serviços. Cad Saude Publica 1998; 14(2):355-365.

6. Vaitsman J, Andrade GRB. Satisfação e responsividade: formas de medir a qualidade e a humanização da assistência à saúde. Cien Saude Colet 2005; 10(Supl. 3):599-513.

7. Aragon SJ. Commentary: a patient-centered theory of satisfaction. American J of Med Quality 2003; 18(Supl. 6):225-228.

8. Aragon SJ, Gesell SB. A patient satisfaction theory and its robustness across gender in emergency departments: a multigroup structural equation modeling investigation. American J of Med Quality 2003; 18(Supl. 6):229-241.

9. Minayo MCS, Assis SG, Souza ER, organizadores. Avaliação por triangulação de métodos: abordagem de programas sociais. Rio de Janeiro: Editora Fiocruz; 2005.

10. Assis SG, Deslandes SF, Minayo MCS, Santos NC. Definição de objetivos e construção de indicadores visando à triangulação. In: Minayo, MCS, Assis SG, Souza ER, organizadores. Avaliação por triangulação de métodos: abordagem de programas sociais. Rio de Janeiro: Editora Fiocruz; 2005. p. 105-132.

11. Minayo MCS. O desafio do conhecimento: pesquisa qualitativa em saúde. São Paulo: Hucitec; 2006.

12. Instituto Brasileiro de Geografia e Estatístico (IBGE). Censo Demográfico 2000. [Site na Internet]. [acessado $2009 \mathrm{fev}$ 12]. Disponível em: http:// www.ibge.gov.br/censo/quest_amostra.pdf

13. Gomes R. Análise e interpretação de dados de pesquisa qualitativa. In: Minayo MCS, organizadora. Pesquisa social: teoria, método e criatividade. Petrópolis: Vozes; 2007. p 79-108.

14. Ayres JRCM. Cuidado e humanização das práticas de saúde. In: Deslandes S, organizadora. Humanização dos cuidados em saúde: conceitos, dilemas e práticas. Rio de Janeiro: Fiocruz; 2006. p. 49-83.
15. Atkinson SJ. Anthropology in research on the quality of health services. Cad Saude Publica 1993; 9(Supl. 3):283-299.

16. Franco SC, Campos CWS. Avaliação da qualidade de atendimento ambulatorial em pediatria em um hospital universitário. Cad Saude Publica 1998; 14(Supl. 1):61-67.

17. Turrini RNT, Lebrão ML, Cesar CLG. Resolutividade dos serviços de saúde por inquérito domiciliar: percepção do usuário. Cad Saude Publica 2008; 24 (Supl. 3):663-674.

18. Gouveia GC, Souza WV, Luna CF, Souza-Junior, PRB, Szwarcwald CL. Health care users' satisfacton in Brazil 2003. Cad Saude Publica 2005; 21(Supl. 1):109-118.

19. Silva AMR, Andrade SM, Thomsom Z. Opiniões de mães de crianças que morreram no primeiro ano de vida no Município de Londrina, Paraná, Brasil. Cad Saude Publica 2002; 18(5):1295-1302.

20. Bós AMG, Bós AJG. Determinantes na escolha entre atendimento de saúde privada e pública por idosos. Rev Saude Publica 2004; 38(Supl. 1):113-120.

21. Farias LO. Estratégias individuais de proteção à saúde: um estudo da adesão ao sistema de saúde suplementar. Cien Saude Colet 2001; 6(2):405-416.

22. Brasil. Ministério da Saúde (MS). Programa Nacional de avaliação dos Serviços: Resultado do processo avaliativo 2004 -2006. Brasília: Ministério da Saúde; 2007.

23. Traverso-Yépez M, Morais NA. Reivindicando a subjetividade dos usuários da Rede de Saúde: para uma humanização do atendimento. Cad Saude Publica 2004; 20(1):80-88.

24. Gomes R. Sexualidade masculina, gênero e saúde. Rio de Janeiro: Fiocruz; 2008.

25. Caprara A, Franco ALS. Relação médico-paciente e humanização dos cuidados em saúde: limites, possibilidades e falácias. In: Deslandes, SF, organizadora. Humanização dos cuidados em saúde: conceitos, dilemas e práticas. Rio de Janeiro: Fiocruz; 2006. p. 85-88.

Artigo apresentado em 02/03/2009

Aprovado em 07/05/2009

Versão final apresentada em 08/05/2009 
